\title{
Improving computer simulation of vacuum pumping process of the plasma sputtering system
}

\section{$\underline{\text { Roman Sheremeta }}$}

IMIT, Lviv Polytechnic National University, UKRAINE, Lviv, 12 Bandera street, E-mail: r1sheremeta@gmail.com

Abstract - The aim of the research is to improve mathematical model of the computer simulation of the vacuum pumping process. This is implemented by introducing additional blocks of operators, using of which allows to achieve optimum modes of carrying out the technological process of vacuum sputtering of $\mathrm{SiO}_{2}$ on polymeric surfaces.

Keywords - sputtering, vacuum sputtering, silicon dioxide, vacuum system, mathematical model, calculation, computer simulation.

\section{Introduction}

Modern packaging standards require the use of the latest technologies to improve the quality of transportation and long-term storage of bulk products and beverages. One of the ways to solve this problem is to spray thin protective coatings on the inner surface of the plastic container. In particular, the technology of vacuum plasma coating with silicon dioxide on the inner surface of PET containers has been developed. Taking into account the repeatability of the process, there is a need to ensure that fastest possible pumping out of the vacuum plasma sputtering system is achieved. As well as to determine that the optimal amount of activating gas mixture is injected to create the required partial pressure in the working chamber.

\section{Research Description}

In the publication [1], a multifactor mathematical model describing the process of vacuum pumping of gases from closed systems was created. In the publication [2], this model became universalized, which allowed to present a wide range of design features of real vacuum systems. This paper also proposes computer simulation for calculating the parameters of pumping out vacuum systems in the Simulink environment from the Matlab software package. Example of vacuum pumping process of the plasma sputtering system simulation is shown in Fig. 1.

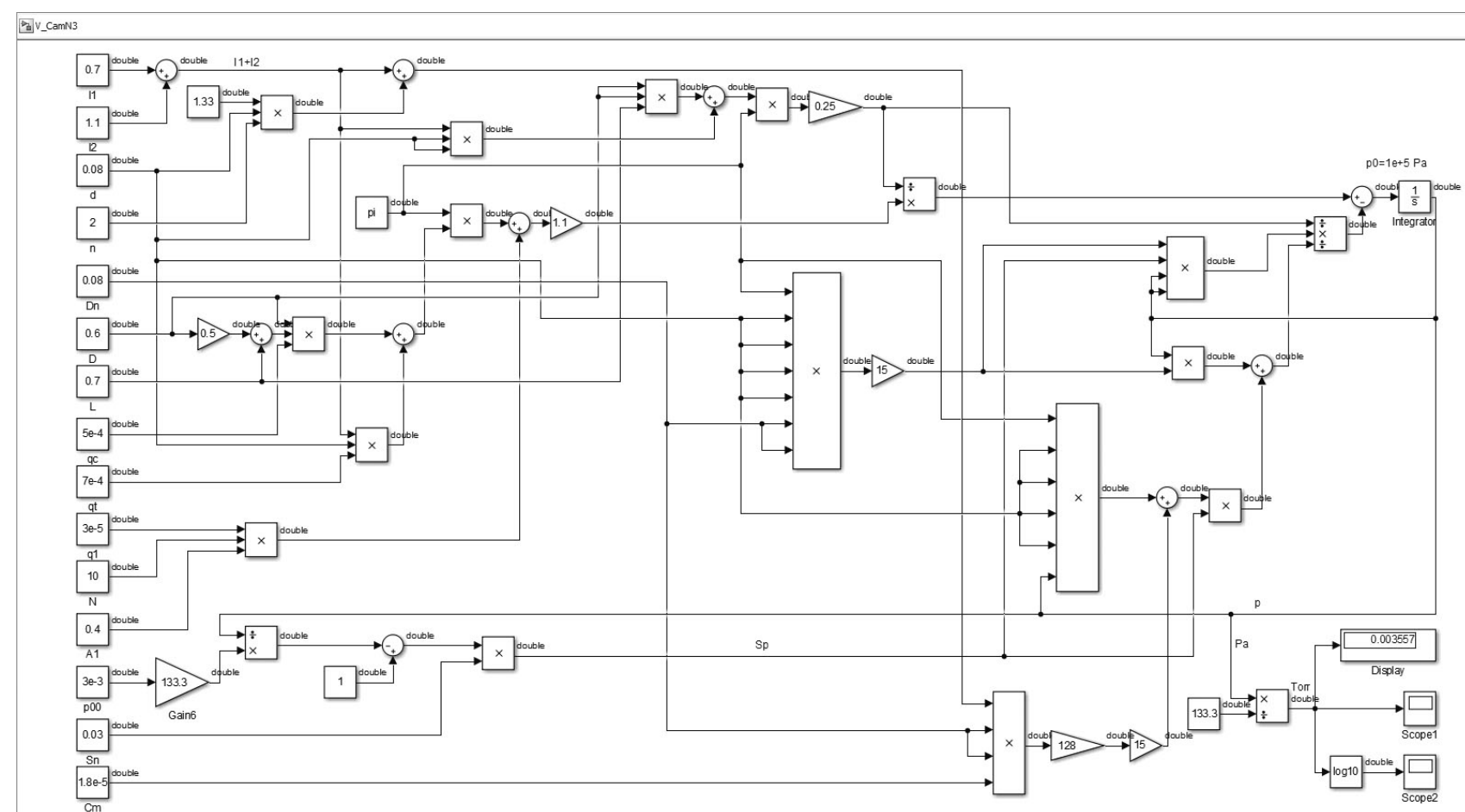

Fig. 1. Example vacuum pumping process of the plasma sputtering system simulation 
The proposed simulation allows to analyze the gas state in any structural element of the vacuum system during its operation. The graphical dependence of the pressure change in the working chamber of the system during the achievement of a given vacuum is shown in Fig. 2.

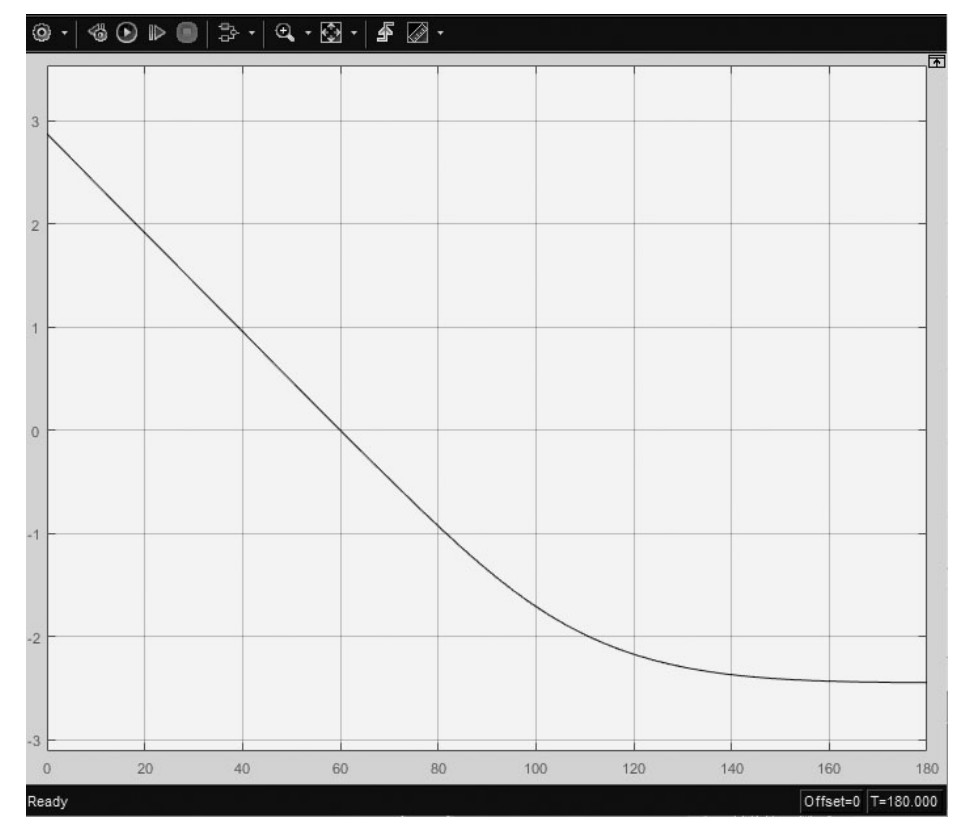

Fig. 2. Change of pressure in the working chamber of the system while achieving operational state

In order to automate calculation of the optimal pumping time, the simulation has been improved. To do this, a programmable timer and a comparator were introduced into the general simulation. With this innovation, when simulating the process of pumping out vacuum system a comparison of the running gas pressure in the working chamber is compared with the specified. In case of equality of these values timer indication is being fixed while signaling the moment of achieving of the working pressure in the vacuum system. The results of the computer simulation of the pumping out process showed sufficient accuracy compared to the experimentally obtained results.

\section{Conclusions}

Mathematical modeling and computer simulation of the pumping process allows to make optimal cyclograms of the individual elements of the vacuum system of technological installations. One of the results of the study is the creation of automated system that allows calculation of the parameters of the pumping process of vacuum systems, which should be used in the design and operation of this equipment.

\section{References}

[1] R. M. Sheremeta, (2001). Calculation of the kinetics of the process of forvacuum pumping of the vacuum system. Publication of IX Ukrainian - Polish Conference on "CAD in Machinery Design. Implementation and Educational Problems". Warsaw, Poland p. 131136.

[2] R. M. Sheremeta, Z. A. Stotsko, S. Plaska, (2008). Automated Calculation of Process Bailing of Vacuum Systems. Publication of the XVI Ukrainian - Polish Conference on "CAD in Machinery Design. Implementation and Educational Problems". Lviv, Ukraine.

[3] R. M. Sheremeta, (2011). Computer simulation of the vacuum systems pumping technological process. Lviv Polytechnic National University bulletin №713 "Optimization of production processes and technical control in mechanical engineering and instrument making", p. 132-137. 